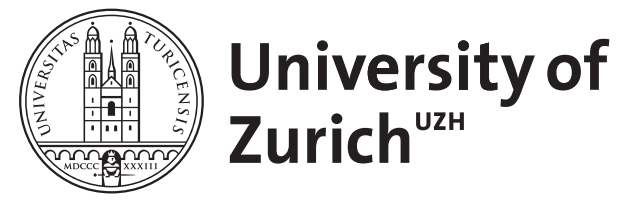

Zurich Open Repository and Archive

University of Zurich

University Library

Strickhofstrasse 39

CH-8057 Zurich

www.zora.uzh.ch

Year: 2014

«Vielfalt statt Einfalt»-Validierte Tipps für den hausärztlichen Praxisalltag

Héritier, F G ; Rosemann, T

Posted at the Zurich Open Repository and Archive, University of Zurich

ZORA URL: https://doi.org/10.5167/uzh-95070

Journal Article

Published Version

Originally published at:

Héritier, F G; Rosemann, T (2014). «Vielfalt statt Einfalt» - Validierte Tipps für den hausärztlichen Praxisalltag. PrimaryCare, 14(2):23-24. 


\title{
«Vielfalt statt Einfalt» - Validierte Tipps für den hausärztlichen Praxisalltag
}

\author{
SwissFamilyDocs Conference 2014 , 28. - 29. August in Zürich
}

\begin{abstract}
Am 28. und 29. August 2014 findet im Kongresshaus Zürich die 4. SwissFamilyDocs Conference («Vielfalt statt Einfalt») statt. Organisiert wird der von der SGAM und den fünf universitären Schweizer Hausarztinstituten (IHAM) getragene Fortbildungskongress mit dem Schwerpunkt auf Allgemeine Innere Medizin und Hausarztmedizin turnusgemäss vom Institut für Hausarztmedizin der Universität Zürich. Referenten aus dem In- und Ausland geben einen Überblick über Perspektiven und Trends in vielen für die Hausarztmedizin relevanten Bereichen, einschliesslich der personalisierten Medizin. Das Vorprogramm steht auf der Kongresswebsite zum Download als PDF bereit, das detaillierte Programm wird ausserdem fortlaufend aktualisiert (www. swissfamilydocs.ch/2014). Abstracts können bis am 20. April 2014 eingereicht werden.
\end{abstract}

Die rasant zunehmenden diagnostischen und therapeutischen Möglichkeiten, die Flut neuer wissenschaftlicher Erkenntnisse und erneut angepasster Expertenempfehlungen, der vermehrte zu berücksichtigende Patientenwunsch und nicht zuletzt die Verpflichtung zur Kostendämpfung machen eine hinsichtlich aller Erwartungen «vernünftige» Entscheidungsfindung gerade für uns Hausärztinnen und Hausärzte zu einer grossen Herausforderung. Mit dem Tagungsmotto «Vielfalt statt Einfalt» möchte die SwissFamilyDocs Conference 2014 einerseits ein wenig Orientierung geben im Dickicht der medizinischen Möglichkeiten. Das Thema bietet aber auch eine gute Gelegenheit, über einen kritischeren, verstärkt evidenzbasierten und gleichzeitig kreativeren Einsatz des medizinisch Möglichen und der vorhandenen Ressourcen im Alltag nachzudenken. Oft erscheint es sinnvoll, neue Wege zu beschreiten und hier und da gewohnte Pfade zu verlassen. Doch jeweils welche?

\section{Weniger ist oft mehr}

Als Eröffnungsvorlesung wird uns Prof. Thomas Bodenheimer vom Department of Family and Community Medicine der Medical School der University of California in San Francisco (UCSF) zum Thema «Medical home concept» die Erfahrungen US-amerikanischer Kollegen mit einem Praxisorganisationskonzept schildern. In der ersten Keynote Lecture geht Prof. Milo Puhan, Zürich, der Frage nach, warum Metaanalysen für eine evidenzbasierte Prävention heutzutage nicht ausreichen. Dass es beim Ruf nach mehr Vielfalt nicht darum geht, blindlings Bewährtes durch Neues zu ersetzen, nur weil es «in» ist, will uns Prof. Nicolas Rodondi, Bern, in seiner Keynote Lecture «Des examens inutiles - du complexe au simple» und darüber hinaus in einem Workshop aufzeigen. Denn wohlüberlegte Entscheidungen vorausgesetzt, lässt sich im Sinne von «less is more» häufig ein eindeutiger «Mehr»-Wert erzielen.

\section{Bewährte Vielfalt an Lernformaten}

Die SwissFamilyDocs Conference wartet insgesamt mit 12 Seminaren und 26 Workshops auf, die allesamt von einem Referentenduo aus Hausarzt und Spezialist präsentiert werden. Bei den Seminaren wurde neu eine Kategorisierung betreffend der Breite des Fokus oder ihrer Zukunftsgerichtetheit vorgenommen (s. Kasten). Des Weiteren umfasst das Programmangebot freie Mitteilungen, «Meet the Keynote Lecturer»-Sessions, «Latest News»-Sessions, «Diamonds»-Sessions mit Fallbeispielen aus der Praxis, «Refresher» und natürlich «Skill Labs» für das Verfeinern praktischer und kommunikativer ärztlicher Fertigkeiten. Abgerundet wird das Programm durch die Verleihung des IHAMB-Forschungspreises der Sandoz für die besten wissenschaftlichen Abstracts, durch zahlreiche Satellitensymposien sowie durch die Political Arena.

\section{Kompaktlehrgang "Good Clinical Practice» als Novum} Neu ist 2014 der Kompaktlehrgang "Good Clinical Practice» mit seinem eintägigen Modul 1. Der von der SAMW getragene, bei der Swissmedic akkreditierte Lehrgang richtet sich an Haus- und Kinderärztinnen, die sich als Zentrum an klinischen Studienprojekten beteiligen wollen. Der von Dr. Markus Gnädinger, Steinach, moderierte Lehrgang wird inhaltlich von PD Dr. Jürg Lustenberger, Leiter Quality Management am Clinical Trials Center des Universitätsspitals Zürich und CEO von SwissPharmAudit, bestritten. Das aus den Teilen A und B bestehende Modul 1 vermittelt neben rechtlichethischen Grundlagen (ICH-GCP-Richtlinien) Kenntnisse zu Studiendesign und -protokoll, Datenmanagement (Erfassung, Aufbewahrung), zum Vorgehen bei unerwünschten Arzneimittelwirkungen sowie zum Thema Qualitätssicherung mit der Bedeutung von Monitoring und Audits und bei klinischen Studienvorhaben.

Der Lehrgang wird mit identischen Inhalten sowohl am KHM-Kongress (26. Juni 2014) als auch an der SFD Conference (28. August 2014) angeboten, wobei die Modulteile A und B für denselben Kongress oder separat gebucht werden können.

\section{Erneut dabei:}

Lehrgang «Kooperative Führung für Hausärzte»

Mit der Frage, wie sich angesichts wachsender Interdisziplinarität und -professionalität Behandlungsziele im Zusammenspiel mit verschiedenen Berufsgruppen erreichen lassen, befasst sich der praxisorientierte Lehrgang "Kooperative Führung für Hausärzte» mit den Psychologen und Coaching-Experten Prof. Christoph Clases und Prof. Theo Wehner als Moderatoren. Der Lehrgang wird auf- 
grund der positiven Resonanz und der grossen Nachfrage im letzten Jahr erneut durchgeführt und besteht auch 2014 aus zwei Kurstagen (1. Teil KHM-Kongress 2014, 2. Teil SFD-Kongress 2014) sowie zwei Teilen zum Selbststudium.

In den 2 Kursmodulen erhalten die teilnehmenden Haus- und Kinderärzte Gelegenheit, ihre eigenen «Führungs- und Kooperationsbeziehungen» mit anderen in die Grundversorgung involvierten Gesundheitsberufen zu diskutieren und den Umgang mit spannungsgeladenen und konfliktbehafteten Situationen zu erlernen, wie sie aufgrund unterschiedlicher Vorstellungen und Konzepte durch verschiedenartigen Erfahrungs- und Ausbildungshintergründe schnell entstehen. Informationen zur Anmeldung und Verfügbarkeit einzelner Kurse finden Sie auf der Kongress-Website (www.swissfamilydocs.ch/2014).

\section{SwissFamilyDocsConference: ein Kongress nicht nur für Hausärzte}

Auch wenn sich der SFD-Kongress in erster Linie an die Hausärztinnen und Hausärzte sowie an die Kinderärztinnen und Kinderärzte in der Schweiz richtet, sind auch Assistenzärztinnen und Assistenzärzte sowie Studierende mit Weiterbildungsziel Hausarztmedizin sowie generell alle Jungärztinnen und -ärzte, deren künftiges Arbeitsgebiet noch offen ist, eingeladen.

Willkommen heissen wir aber auch all diejenigen Kolleginnen und Kollegen, die sich einfach für das breite Spektrum der Hausarztmedizin interessieren. Die Kongressteilnahme lohnt sich auch deswegen, weil auch das diesjährige Programm erneut zahlreiche Schnittstellen mit dem stationären Sektor des Gesundheitssystems aufweist.

Reservieren Sie sich am besten schon jetzt den Termin der 4. SwissFamilyDocs Conference - oder noch besser: melden Sie sich an. Wir freuen uns auf einen vielfältigen Kongress und vor allem auf den Gedankenaustausch mit Ihnen!
Fit sein für die Praxis: Future-, Up-to-Dateund State-of-the-Art-Seminare

Während an der SwissFamilyDocs Conference in den «FutureSeminaren» entweder zukünftige Therapiemöglichkeiten (Endoluminaler Aortenklappenersatz, Knorpelersatzforschung) oder bevorstehende Herausforderungen der Grundversorgung (Life-Coach-Approach zur Gesundheitsberatung Jugendlicher, Personalisierte Medizin in der Hausarztmedizin) diskutiert werden, liefern «Up-to-Date-Seminare» und «State-of-the-Art-Seminare» einen Überblick über neueste diagnostische oder therapeutische Entwicklungen bzw. greifen einzelne aktuelle klinische Themen der Allgemeinen Inneren Medizin und der Hausarztmedizin heraus - darunter Diabetes, Infektiologie, MSBehandlung, Osteoporose, Palliativpflege, Pneumologie und Schmerzen als Ausdruck einer dissoziativen Störung. Die 26 Workshops decken neben vielen relevanten medizinischen Themen auch spannende organisatorische Fragestellungen der Hausarztmedizin ab. Hierzu zählen "Mitarbeiterrekrutierung», «Skillmix konkret: der Einsatz von Advanced Nursing Practices in Ärztenetzwerken» oder «Praxislabors zur Steuerung des Antibiotika-Einsatzes»

\section{Korrespondenz:}

Dr. med. François-Gérard Héritier

Präsident SGAM

Mitglied des Organisationskomitees der SwissFamilyDocs Conference 2013 heritier.vf[at]vtxnet.ch

Prof. Dr. med. Thomas Rosemann, PhD

Ordinarius für Hausarztmedizin

Direktor des Institutes für Hausarztmedizin Zürich thomas.rosemann[at]usz.ch 